Tanja Lindacher \& Kathrin Dedering

\title{
Unterrichtliche Kooperation zwischen Professionellen an inklusiven Schulen Überblick zum Forschungsstand
}

\section{Zusammenfassung}

Mit Blick auf ein spezifisches inklusives Bildungsangebot - den Unterricht - und die Kooperation der in ihn involvierten Professionellen nimmt der Beitrag knapp zehn Jahre nach Inkrafttreten der UN-Behindertenrechtskonvention eine aktuelle empirische Bestandsaufnahme vor. Er fasst Befunde zu der faktischen Ausgestaltung der unterrichtlichen Kooperation, zu Einstellungen und Bewertungen der Professionellen bezüglich Kooperation und zur Wahrnehmung von Kooperation aus dem Blickwinkel der Schülerschaft zusammen und entfaltet zwei Dimensionen, die für die Weiterentwicklung unterrichtlicher Kooperation zentrale Herausforderungen darstellen.

Schlüsselwörter: Kooperation, inklusiver Unterricht, inklusive Bildung, Professionalisierung

\section{Co-Teaching in Inclusive Classrooms}

\section{A Literature Review}

\section{Summary}

Focusing on co-teaching in inclusive classrooms as a specific instrument of inclusive education, the article reviews - almost ten years after the implementation of the United Nations Convention on the Rights of People with Disabilities - current empirical research. It summarizes findings referring to the realization of co-teaching, to corresponding attitudes and views expressed by professionals, and to the perception of co-teaching by pupils. Finally, two dimensions relevant to its further development are outlined. Keywords: co-teaching, inclusive classrooms, inclusive education, professionalization 


\section{Einführung}

Seit der Unterzeichnung der UN-Behindertenrechtskonvention im Jahre 2009 sieht sich Deutschland mit der Notwendigkeit konfrontiert, ein inklusives Bildungssystem bereitzustellen, das dazu beiträgt, das Recht von Menschen mit Behinderungen auf Bildung ohne Diskriminierung und auf der Grundlage der Chancengleichheit zu verwirklichen.

Dabei wird der Arbeit von Akteuren unterschiedlicher beruflicher Provenienz, die an der Bereitstellung eines inklusiven Bildungsangebotes mitwirken, in Literatur und Praxis eine große Bedeutung beigemessen: Das aufeinander bzw. miteinander abgestimmte Vorgehen - die Kooperation - der Professionellen ${ }^{1}$ wird als innerorganisationale Struktur mit stützender Wirkung und als Gelingensbedingung für die Umsetzung von Inklusion betrachtet (Werning, 2014).

Der vorliegende Beitrag richtet seinen Blick auf ein spezifisches inklusives Bildungsangebot - den Unterricht - und die in ihn involvierten Professionellen - Regelschullehrkräfte, Sonder-/Förderschullehrkräfte, Sozialpädagog*innen, Vertreterinnen außerschulischer Einrichtungen etc. Vor dem Hintergrund der herausgestellten Relevanz von Kooperation geht er der Frage nach, was wir knapp zehn Jahre nach Inkrafttreten der UN-Konvention über die unterrichtliche Kooperation der pädagogischen Professionen an inklusiven Schulen wissen. Auf der Basis einer systematischen Sichtung der derzeitigen empirischen Befundlage nimmt er eine aktuelle Bestandsaufnahme vor. Darüber hinaus formuliert er aus unserer Sicht wesentliche Desiderate einer zukünftigen Forschung zur unterrichtlichen Kooperation von Professionellen.

\section{Begriffliche Zugänge}

Unterrichtliche Kooperation wurde bereits im Kontext der Integrationspädagogik (Haeberlin, Jenny-Fuchs \& Moser Opitz, 1992) und im Rahmen der schulischen Ganztagsversorgung (Ditzinger, Fussangel \& Böhm-Kasper, 2011) intensiv diskutiert und gilt gemeinhin als Qualitätsmerkmal guter Schulen (z.B. Steinert, Klieme, Maag Merki, Döbrich, Halbheer \& Kunz, 2006). Allerdings mangelt es bis heute an einer konsistenten und verbindlichen Begriffsverwendung (Ahlgrimm, Krey \& Huber, 2012).

1 Um zu unterstreichen, dass Kooperationen von zwei oder mehr Beteiligten mit eher unterschiedlicher beruflicher Provenienz getragen werden, sprechen wir von Kooperationen zwischen Professionellen - wenngleich im Einzelnen zu prüfen ist, inwiefern Professionalisierung vorliegt (Stichweh, 1992). 
Vangrieken, Dochy, Raes \& Kyndt (2015) dokumentieren anhand eines Literaturreviews die Vielfalt an Begriffen, die mit „teacher collaboration“ assoziiert werden. Sie unterstreichen, dass „collaboration“ kein klar definiertes und statisches Konzept darstellt, sondern sich in verschiedenen Ausprägungsformen auf einem Kontinuum zwischen „completely individualised teacher work and [...] collaborative work“ (ebd., S.25) verorten lässt. Marvin (1990, S.41) unterscheidet vier Stufen der Zusammenarbeit: „coactivity“, „cooperation“, „coordination“ und „collaboration“. Dabei gilt „collaboration“ als die effektivste und gleichzeitig am schwierigsten $\mathrm{zu}$ realisierende Form von Zusammenarbeit, da sie neben einer grundlegenden Übereinstimmung in den Werthaltungen der Beteiligten auch eine gemeinsam getragene Unterrichtsvorbereitung und Unterrichtsreflexion impliziert. In Abgrenzung hierzu signalisiert „cooperation“ einen deutlich geringeren Grad an Verbindlichkeit (vgl. ebd.).

Einen Überblick über Formen unterrichtlichen Zusammenwirkens, sogenannte „co-teaching approaches“, die sich auf zwei in einem paritätischen Verhältnis zueinander stehende Lehrende beziehen, geben Friend, Cook, Hurley-Chamberlain \& Shamberger (2010). Sie differenzieren je nach Aufgabenübernahme zwischen „One teach, one observe“, „Station Teaching“, „Parallel Teaching“, „Alternative Teaching“, „Teaming“ sowie „One teach, one assist“ (ebd., S. 12). Innerhalb dieser Formen wechseln die Lehrenden idealiter flexibel ihre Rolle (vgl. ebd., S. 13).

In der deutschsprachigen Literatur lassen sich drei begriffliche Zugänge zur unterrichtlichen Kooperation unterscheiden:

Ein erster Zugang betont Fragen der Ausprägung oder Qualität von Kooperation und lehnt sich an Niveaustufen an (z. B. Rolff \& Steinweg, 1980; Steinert et al., 2006). Exemplarisch sei hier auf Gräsel, Fussangel \& Pröbstel (2006) verwiesen, die auf der Basis einer organisationspsychologischen Definition von Kooperation drei Formen der Zusammenarbeit von Lehrkräften unterscheiden: 1.wechselseitiger Austausch von Informationen und Materialien, 2.arbeitsteilige Kooperation, die eine verteilte Bearbeitung von Aufgaben vorsieht, 3. Kokonstruktion im Sinne gemeinsamer Aufgaben- oder Problemlösungen (vgl. ebd., S. 209-210). Die Modellierungen erlauben es, unterschiedliche Kooperationsniveaus unter Regelschullehrkräften zu identifizieren, klammern jedoch die strukturellen Aspekte, die mit der unterrichtlichen Kooperation von zwei oder mehr Professionellen einhergehen, aus.

Sich von Niveaugruppen oder Qualitätsindikatoren distanzierend fokussiert der zweite Zugang zum Kooperationsbegriff den prozessualen Charakter von Kooperation. So konzipiert etwa Kullmann (2010) vor dem Hintergrund eines organisationspsychologischen Verständnisses von Kooperation ein Wirkungsmodell, das aus drei Etappen besteht: 1. Zielartikulation und Verbesserung des Planungshandelns, 2. mehrfache Erprobung und Evaluation der veränderten Planung, 3. Prozessierung und 
Verbesserung der Kompetenzen der Schülerschaft (vgl. ebd., S. 88). Kullmanns Modell verdeutlicht zwar die Relevanz rekursiver Prozesse für eine nachhaltige Verbesserung des unterrichtlichen Handelns, nimmt allerdings keinen Bezug auf die Integration sonderpädagogischer Expertise.

Im Fokus des dritten Zugangs, der u.a. das Verhältnis von Regelschul- und Sonderschullehrkräften aufgreift, steht die Annahme, dass die Anerkennung unterschiedlicher Handlungslogiken von Professionen für Kooperation konstituierend sei (z.B. Willmann, 2009). Chilla (2012) fasst Kooperation als Leitgedanken inklusiver Bildung und bezieht sie auf die Unterstützung der Bildungs- und Erziehungsprozesse von Schüler*innen. Insofern sieht sie Kooperation nicht an eine Zuweisung sonderpädagogischen Förderbedarfs gekoppelt, sondern als individuelle Entwicklungsaufgabe von Lehrenden, die eine Auseinandersetzung mit sich selbst und dem Gegenüber erfordert (vgl. ebd., S. 115; Lütje-Klose \& Urban, 2014, S. 121).

Der Begriff der unterrichtlichen Kooperation ist nicht mit jenem der Professionellen Lerngemeinschaften gleichzusetzen: Unterrichtliche Kooperation stellt eine technische Gelingensbedingung für Professionelle Lerngemeinschaften dar; deren Bestimmungskriterien reichen jedoch weit über Kooperation hinaus (vgl. Bonsen \& Rolff, 2006, S. 179). Das skizzierte Spektrum an begrifflichen Zugängen zeigt, dass die Thematisierung von unterrichtlicher Kooperation aus unterschiedlichen Perspektiven und mit verschiedenen Zielsetzungen erfolgt.

\section{Befunde zur unterrichtlichen Kooperation zwischen Professionellen}

Die in Kapitel 2 thematisierten unterschiedlichen Begriffsvarianten zeigen sich auch in den Befunden zur unterrichtlichen Kooperation zwischen Professionellen im Kontext inklusiver Schulen. Für eine systematisierte Darstellung des Forschungsstandes orientieren wir uns an der Frage, wie die faktische Ausgestaltung beschrieben wird (Kap. 3.1), welche Einstellungen zu und Bewertungen von Kooperation berichtet werden (Kap. 3.2) und wie Kooperation aus dem Blickwinkel der Schülerschaft wahrgenommen wird (Kap. 3.3).

\subsection{Ausgestaltung von unterrichtlicher Kooperation}

Ein Großteil der Publikationen zur unterrichtlichen Kooperation zwischen Professionellen konzentriert sich auf die beiden Berufsgruppen der Regel- und Sonderschullehrkräfte. Dabei geht es empirisch schwerpunktmäßig um die Frage, wie „co-teaching“ im inklusiven Unterricht von den beiden Professionen ausgestal- 
tet wird. Aspekte der Verteilung von Zuständigkeiten bzw. der Rollenübernahme stehen im Mittelpunkt. Häufig wird von einem nicht gleichwertigen Status der Sonderschullehrkraft berichtet. Ihre Aufgabe bestehe vor allem darin, Schüler*innen mit besonderem Förderbedarf (gegebenenfalls räumlich separiert) zu fördern und/ oder beobachtend zu begleiten. Die Regelschullehrkräfte fungierten als Expert*innen für das jeweilige Thema, während die Sonderschullehrkräfte eine unterstützende oder zuarbeitende Position einnähmen und somit ihre sonderpädagogische Expertise kaum zur Anwendung bringen könnten (vgl. Weiss \& Lloyd, 2002, S. 67). Umgekehrt thematisieren Sonderschullehrkräfte bei der Übernahme von Fachunterricht ohne Doppelbesetzung einen Verlust ihrer sonderpädagogischen Sichtweise (vgl. Arndt \& Werning, 2016, S. 170).

Diese Befunde werden durch eine Metaanalyse von Scruggs, Mastropieri \& McDuffie (2007) gestützt. Im Rahmen von „co-teaching" wird am häufigsten eine Version von "one teach, one assist" praktiziert, bei der die Sonderschullehrkraft eine untergeordnete Rolle einnimmt. Dies gilt sowohl für die Primar- als auch für die Sekundarstufe, wenngleich sich der geringere Status von Sonderschullehrkräften konsistenter im Sekundarbereich abzeichnet (vgl. ebd., S. 408). Wenig erforscht sind in diesem Zusammenhang die beiden Formen „team teaching“ und „station teaching“. Gurgur \& Uzuner (2011) richten hierauf ihre Aufmerksamkeit und benennen umfangreiche Herausforderungen in den vor- und nachbereitenden Unterrichtsaktivitäten von Regel- und Sonderschullehrkräften.

Eine überwiegende Zuständigkeit von Sonderschullehrkräften für Lernende mit sonderpädagogischem Förderbedarf wird von Kreis, Wick \& Kosorok Labhart (2014) insofern relativiert, als es bei der Umsetzung von Förderzielen gegebenenfalls situativer Rollenklärungen auf der unterrichtlichen Mikroebene bedürfe (vgl. ebd., S. 345346). Die von Regelschullehrkräften berichteten Aktivitätenrepertoires belegen, dass systematische Diagnostik und Abklärung nicht nur im Zuständigkeitsbereich von Sonderschullehrkräften liegen, sondern auch prototypisch für die eigene Unterrichtstätigkeit sind (vgl. Kreis, Wick \& Kosorok Labhart, 2016, S. 155).

Wie Kooperation ausgestaltet wird, ist darüber hinaus auch von den Vorstellungen von individueller Förderung abhängig (vgl. Arndt \& Werning, 2016, S. 170). Diesbezüglich konstatieren Baumann, Henrich \& Studer (2013) eine Interaktion der Qualität der unterrichtsbezogenen Kooperation mit der Qualität der Umsetzung einzelner Aspekte von Differenzierung (vgl. ebd., S. 47-48).

Der inhaltliche Austausch von Regel- und Sonderschullehrkräften bezieht sich vor allem auf sozial-emotionale Aspekte der Förderung (vgl. Pool Maag \& Moser Opitz, 2014, S. 140). Ebenfalls Gegenstand der Kommunikation sind der Leistungsstand der Schülerschaft, die Unterrichtsthemen sowie organisatorische Angelegenheiten. Wenig werden hingegen Fragen der Didaktik, Diagnostik und Zusammenarbeit im Team 
thematisiert (vgl. Gebhard et al., 2014, S. 22). Dieser Befund steht im Widerspruch zu den bei Kreis et al. (2016) generierten Befunden, wonach für Regelschullehrkräfte die Gestaltung von Lerngelegenheiten eine prototypische Aktivität ist und daher auch mit Absprachen einhergeht (vgl. ebd., S. 148).

Vereinzelt wird bei der Wahl des Förderortes und der Gestaltung von Fördermaßnahmen auf Spannungsfelder verwiesen, die sich aus Stigmatisierungseffekten, selbstwertschwächenden Leistungsvergleichen innerhalb der Klassengemeinschaft sowie den finanziellen Ressourcen der Schulen ergeben (vgl. Pool Maag \& Moser Opitz, 2014, S. 140). Neben Tendenzen zur äußeren Differenzierung mit Hilfe von Förderräumen wird auf folgende Merkmale der Ausgestaltung von Kooperation hingewiesen: fehlende Kooperations- und Vorbereitungszeiten außerhalb des Unterrichts und eine unzureichende strukturelle Einbindung von Kooperation als professioneller Arbeitsform in die Organisation von Schule (vgl. Arndt \& Werning, 2013, S. 35).

Die unterrichtliche Kooperation zwischen Lehrkräften und dem weiteren pädagogischen Personal ist bislang nur ansatzweise untersucht. Sie ist aufgrund der ungeklärten Bestimmung des Verhältnisses zwischen prekären Beschäftigungsverhältnissen von Para-Professionellen, z. B. Integrationshelfer*innen, und den Lehrkräften an einer Schule besonders komplex (vgl. Heinrich \& Lübeck, 2013, S. 106; zur Schulsozialarbeit vgl. Fabel-Lamla \& Reinecke-Terner, 2015, S. 154).

\subsection{Einstellungen zu Kooperation und Bewertungen von Kooperation}

Mit Blick auf Inkonsistenzen bezüglich der Übernahme von Verantwortung unter den Beteiligten gehen Stefanidis \& Strogilos (2015) der Frage nach, inwiefern sich die Einstellungen von Sonder- und Regelschullehrkräften hinsichtlich ihrer jeweiligen Zuständigkeiten während und außerhalb des gemeinsamen Unterrichts unterscheiden. Sie kommen zu dem Ergebnis, dass sich beide Gruppen in ihren Einschätzungen signifikant unterscheiden, d.h., die Sonderschullehrkräfte schätzen die Verantwortung von Regelschullehrkräften für Schülerinnen mit sonderpädagogischem Förderbedarf weitaus höher ein als die Regelschullehrkräfte selbst, sowohl was die Unterrichtsdurchführung und das Verhaltensmanagement als auch vor- und nachbereitende Aktivitäten anbelangt. Ebenso schätzen Sonderschullehrkräfte ihre eigene unterrichtliche und außerunterrichtliche Verantwortung für alle Schüler*innen einer Klasse im Vergleich zu den Regelschullehrkräften höher ein (vgl. ebd., S. 407). Fennick \& Liddy (2001) gelangen hinsichtlich „co-teaching“ zu einem konträren Ergebnis: Sowohl Regelschul- als auch Sonderschullehrkräfte sind der Ansicht, jeweils umfangreichere unterrichtliche Verantwortung innezuhaben als die andere Berufsgruppe (vgl. ebd., S. 229). 
Hellmich, Hoya, Görel \& Schwab (2017) unterstreichen die Relevanz von Einstellungen zur Teamarbeit, von Einstellungen zur Inklusion, von Erfahrungen aus dem integrativen bzw. inklusiven Unterricht sowie von Selbstwirksamkeitsüberzeugungen in Bezug auf die Gestaltung des Unterrichts für die Kooperationsbereitschaft von Lehrkräften. Der Effekt der Erfahrungen auf die Einstellungen der Lehrkräfte zur Inklusion wird dabei durch ihre Selbstwirksamkeitsüberzeugungen mediiert (vgl. ebd., S. 47). Von einem positiven Effekt der Erfahrung oder des erweiterten Wissens im interdisziplinären „team teaching“ auf die Einstellung gegenüber „team teaching“ berichten auch Abegglen, Schwab \& Hessels (2017, S. 437).

Hinsichtlich der Bewertung der Kooperation durch Sonderschul- und Regelschullehrkräfte zeigen sich bedeutsame Unterschiede. So kommen Gebhard et al. (2014) zu dem Ergebnis, dass die Gruppe der Sonderschullehrkräfte die Kooperation in den Bereichen Ziel- und Personenorientierung konsistent negativer bewertet als die Gruppe der Regelschullehrkräfte. Sie führen dies auf eine mangelnde Integration der Sonderschullehrkräfte in die Kollegien der Regelschulen zurück (vgl. ebd., S. 30). Ein weiterer Aspekt für die Bewertung ist der Umfang des Lehrdeputats. Lehrkräfte, die von der ständigen Anwesenheit einer Sonderschullehrkraft ausgehen können, schätzen eine Kooperation signifikant besser ein als ihre Kolleg*innen, bei denen eine Sonderschullehrkraft lediglich stundenweise anwesend ist (vgl. Gebhardt, Schwab, Gmeiner, Ellmeier, Rossmann \& Gasteiger Klicpera, 2013, S. 16). Die Befunde von Schwab, Gebhardt \& Krammer (2015, S. 136) identifizieren Unterschiede zwischen den Schulstufen: In der Primarstufe wird die Kooperation von den beteiligten Lehrkräften positiver eingeschätzt als in der Sekundarstufe.

\subsection{Kooperation aus dem Blickwinkel der Kinder und Jugendlichen}

Nur punktuell wird bei der Analyse unterrichtlicher Kooperation explizit die Perspektive von Schüler*innen einbezogen. Arndt \& Gieschen (2013) weisen auf von Schüler*innen angemerkte Inkonsistenzen zwischen innerer Differenzierung und den Formen der Leistungsbewertung und Leistungsrückmeldung hin (vgl. ebd., S. 60). Schwab (2017) beleuchtet die Wahrnehmung der multiprofessionellen Kooperation durch die Schülerschaft unter der Frage, inwiefern sie mit dem schulischen Wohlbefinden der Schüler*innen sowie mit deren Kooperation untereinander zusammenhängt. Dabei korreliert „team teaching“ von Lehrenden signifikant mit der Kooperation unter Schüler*innen und ihrer emotional-sozialen Inklusion sowie ihrem schulischen Selbstkonzept. Je mehr die Schüler^innen „team teaching“ mögen, desto positiver wirkt sich dies auf ihre eigene Kooperation sowie auf inklusionsorientierte Qualitätsindikatoren aus (vgl. ebd., S. 274). Obwohl die Mehrheit der Befragten sich durch die praktizierte Kooperation gut unterstützt fühlt, bleibt ungeklärt, warum dies für circa ein Drittel der Schüler*innen nicht zutrifft (vgl. ebd., S. 275). 
Embury \& Kroeger (2012) verdeutlichen, dass die praktizierten Formen von Verantwortungsübernahme im Rahmen von "co-teaching“ die Wahrnehmung der Schüler*innen beeinflussen. Während die Sonderschullehrkraft in der einen Klasse in der Form „one teach, one assist“ nur als Hilfsperson wahrgenommen wird, avanciert sie in der anderen Klasse, in der nach der Form „station, parallel, and team teaching“ gearbeitet wird, zu einer als gleichermaßen entscheidungsberechtigt wahrgenommenen Lehrperson (vgl. ebd., S. 109).

\section{Herausforderungen im Zuge von unterrichtlicher Kooperation an inklusiven Schulen}

Die im Überblick zum Forschungsstand referierten Studien lassen erkennen, dass unterrichtliche Kooperation im Kontext der schulischen Umsetzung von Inklusion als Professionalisierungsstrategie unter Lehrkräften zwar eine breite Akzeptanz findet, jedoch mit einem hohen Maß an Kontingenz behaftet ist, so dass es schwierig ist, eindeutige Aussagen über ihre Wirksamkeit zu treffen. In Anlehnung an die in Kapitel 3 erläuterten Befunde sowie unter Berücksichtigung des organisationalen Charakters von Schule und der Spannungsfelder der in ihr tätigen Professionellen werden im Folgenden zwei Dimensionen näher betrachtet, die für die Weiterentwicklung unterrichtlicher Kooperation zentrale Herausforderungen darstellen.

\subsection{Professionell-fachliches Selbstverständnis}

Eine sich bei nahezu allen Studien zum Forschungsstand abzeichnende Frage zielt auf den Umgang mit den Potenzialen verschiedenartiger Expertisen und die damit assoziierten Rollenkonstruktionen. Offensichtlich gelingt es bislang nur unzureichend, spezifisches Professionswissen (hier von Sonderschullehrkräften) im Rahmen unterrichtlicher Kooperation auszuschöpfen und die Verteilung von Zuständigkeiten transparent $\mathrm{zu}$ machen, was erhebliche Auswirkungen auf die jeweils wahrgenommene und praktizierte unterrichtliche Verantwortung hat.

Kunze (2016) tritt dafür ein, die Frage nach der Etablierung von Zuständigkeitsbereichen unterschiedlicher Berufsgruppen nicht polarisierend zugunsten von Ausdifferenzierung oder Entdifferenzierung $\mathrm{zu}$ wenden, sondern stattdessen von einer „Zuständigkeitsdiffusitätsproblematik“ (ebd., S. 273) auszugehen, die neues Licht auf (nicht-)institutionalisierte Zuständigkeiten innerhalb von Kooperationen wirft. Angesichts von sowohl fließenden als auch abgrenzenden Rollenkonstruktionen bleibt die „Frage nach den Möglichkeiten einer Kooperation auf ,Fachkollegen-Niveau““ (Arndt \& Werning, 2016, S. 171) im Mittelpunkt. Es ist daher der Blick darauf zu richten, wie Fachlichkeit zur Sicherung von Unterrichtsqualität für sämtliche Schülerinnen (vgl. 
Laubenstein, Lindmeier, Guthöhrlein \& Scheer, 2015) gestärkt und wie Rollenklärung „als zentrale Professionalisierungsherausforderung im Berufsfeld Schule angesichts von Inklusion“ (Demmer, Heinrich \& Lübeck, 2017, S. 28) auch jenseits von Fortbildungsmaßnahmen verankert werden kann.

\subsection{Systemspezifische Eigenrationalität von Schule und Kooperation}

Unter der Prämisse, dass sich Schule in ihrer Doppelstruktur als Organisations- und Interaktionssystem mit programmatischen Impulsen auf Seiten ihrer Umwelt auseinanderzusetzen und gleichzeitig ihre eigene Systemfunktionalität aufrechtzuerhalten hat, lässt sich unterrichtliche Kooperation in unterschiedlichen Spannungsfeldern verorten. Diese werden zwar vereinzelt thematisiert (z.B. Emmerich \& Werner, 2013), jedoch bedarf es zukünftig einer noch stärkeren empirischen Fokussierung der systemspezifischen Eigenrationalität von Schule und Kooperation, um z. B. verständlich zu machen, warum kooperatives Handeln in inklusiven Schulen einer anderen Systemlogik unterliegt als Kooperation in Einrichtungen der Sozialen Arbeit. Bender \& Heinrich (2016, S. 101) regen in diesem Zusammenhang an, Schwierigkeiten in der Ausgestaltung von Kooperation nicht ausschließlich auf der Ebene von Berufsrollen und Professionsdifferenzen $\mathrm{zu}$ verhandeln, sondern stattdessen von „Strukturproblematiken kooperativer Akteurkonstellationen“ (ebd., S. 101) auszugehen.

\section{Fazit}

Aus dem aktuellen Forschungsstand wird ersichtlich, dass es bislang kaum empirisch fundierte Studien zur Frage nach kooperativen Ausgestaltungsprozessen von und zwischen (Para-)Professionellen im Hinblick auf die Umsetzung des Rechts auf inklusive Bildung an Schulen gibt. Es fehlen Studien, in denen Kooperation sowohl theoretisch-konzeptionell als auch forschungspraktisch als komplexer Forschungsgegenstand auf mehreren Systemebenen ausdifferenziert wird. Ein solcher Ansatz würde der Erkenntnis Rechnung tragen, dass sich ein gesamtgesellschaftlicher Anspruch wie Inklusion nicht einfach an Schule als Organisation delegieren lässt, sondern erst unter spezifischen Entscheidungsprämissen organisationsfähig gemacht werden kann.

\section{Literatur}

Abegglen, H., Schwab, S., \& Hessels, M.G.P. (2017). Interdisziplinäres Teamteaching. Eine empirische Studie über die Einstellung zur Zusammenarbeit von Lehrkräften unterschiedlicher Professionen. Zeitschrift für Pädagogik, 63 (4), 437-455.

Ahlgrimm, F., Krey, J., \& Huber, S.G. (2012). Kooperation - was ist das? Implikationen unterschiedlicher Begriffsverständnisse. In S.G. Huber \& F. Ahlgrimm (Hrsg.), Ko- 
operation. Aktuelle Forschung zur Kooperation in und zwischen Schulen sowie mit anderen Partnern (S. 17-30). Münster: Waxmann.

Arndt, A.-K., \& Gieschen, A. (2013). Kooperation von Regelschullehrkräften und Lehrkräften für Sonderpädagogik im gemeinsamen Unterricht. Perspektiven von Schülerinnen und Schülern. In R. Werning \& A.-K. Arndt (Hrsg.), Inklusion: Kooperation und Unterricht entwickeln (S. 41-62). Bad Heilbrunn: Klinkhardt.

Arndt, A.-K., \& Werning, R. (2013). Unterrichtsbezogene Kooperation von Regelschullehrkräften und Lehrkräften für Sonderpädagogik. Ergebnisse eines qualitativen Forschungsprojektes. In R. Werning \& A.-K. Arndt (Hrsg.), Inklusion: Kooperation und Unterricht entwickeln (S. 12-40). Bad Heilbrunn: Klinkhardt.

Arndt, A.-K., \& Werning, R. (2016). Unterrichtsbezogene Kooperation von Regelschullehrkräften und Sonderpädagog/innen im Kontext inklusiver Schulentwicklung. Implikationen für die Professionalisierung. In V. Moser \& B. Lütje-Klose (Hrsg.), Schulische Inklusion (62. Beiheft der Zeitschrift für Pädagogik) (S. 160-174). Weinheim: Beltz Juventa.

Baumann, B., Henrich, C., \& Studer, M. (2013). Kooperation zwischen Regelschule und Heilpädagogik. In M. Schüpbach, A. Slokar \& W. Nieuwenboom (Hrsg.), Kooperation als Herausforderung in Schule und Tagesschule (S. 35-50). Bern: Haupt.

Bender, S., \& Heinrich, M. (2016). Alte schulische Ordnung in neuer Akteurkonstellation? Rekonstruktionen zur Multiprofessionalität und Kooperation im Rahmen schulischer Inklusion. In V. Moser \& B. Lütje-Klose (Hrsg.), Schulische Inklusion (62. Beiheft der Zeitschrift für Pädagogik) (S. 90-104). Weinheim: Beltz Juventa.

Bonsen, M., \& Rolff, H.-G. (2006). Professionelle Lerngemeinschaften von Lehrerinnen und Lehrern. Zeitschrift für Pädagogik, 52 (2), 167-184

Chilla, S. (2012). Kooperation von Lehrkräften - Standort und Perspektiven. In R. Benkmann, S. Chilla \& E. Stapf (Hrsg.), Inklusive Schule - Einblicke und Ausblicke (S. 103121). Immenhausen: Prolog.

Demmer, C., Heinrich, M., \& Lübeck, A. (2017). Rollenklärung als zentrale Professionalisierungsherausforderung im Berufsfeld Schule angesichts von Inklusion. Zur gegenstandsorientierten Konzeption einer Lehrerfortbildung am Beispiel von Schulbegleitungen. Die Deutsche Schule, 109 (1), 28-42.

Ditzinger, V., Fussangel, K., \& Böhm-Kasper, O. (2011). Interprofessionelle Kooperation an Ganztagsschulen aus der Perspektive der Lehrkräfte - Wie lässt sie sich erfassen und wie wird sie im schulischen Belastungs- und Beanspruchungs-Geschehen bewertet? In K. Speck, T. Olk, O. Böhm-Kasper, H.-J. Stolz \& C. Wiezorek (Hrsg.), Ganztagsschulische Kooperation und Professionsentwicklung. Studien zu multiprofessionellen Teams und sozialräumlicher Vernetzung (S. 114-127). Weinheim: Beltz Juventa.

Embury, D. C., \& Kroeger, S. D. (2012). Let's Ask the Kids: Consumer Constructions of CoTeaching. International Journal of Special Education, 27 (2), 102-112.

Emmerich, M., \& Werner, S. (2013). Kooperationsgestützte Unterrichtsentwicklung zwischen Interventionslogik und schulischer Eigenrationalität. In M. Keller-Schneider, S. Albisser \& J. Wissinger (Hrsg.), Professionalität und Kooperation in Schulen. Beiträge zur Diskussion über Schulqualität (S. 167-184). Bad Heilbrunn: Klinkhardt.

Fabel-Lamla, M., \& Reinecke-Terner, A. (2015). Schulsozialarbeit im inklusiven Schulsystem - Chancen und Herausforderungen der Kooperation in multiprofessionellen Teams. In R. Krüger \& C. Mähler (Hrsg.), Gemeinsames Lernen in inklusiven Klassenzimmern. Prozesse der Schulentwicklung gestalten (S. 147-156). Köln: Carl Link.

Fennick, E., \& Liddy, D. (2001). Responsibilities and Preparation for Collaborative Teaching: Co-Teachers' Perspectives. Teacher Education and Special Education: The Journal of the Teacher Education Division of the Council for Exceptional Children, 24 (3), 229-240. https://doi.org/10.1177/088840640102400307. 
Friend, M., Cook, L., Hurley-Chamberlain, D., \& Shamberger, C. (2010). Co-Teaching: An Illustration of the Complexity of Collaboration in Special Education. Journal of Educational and Psychological Consultation, 20 (1), 9-27. https://doi.org/10.1080/ 10474410903535380.

Gebhard, S., Happe, C., Paape, M., Riestenpatt, J., Vägler, A., Wollenweber, K. U., et al. (2014). Merkmale und Bewertung der Kooperation von Sonderpädagogen und Regelschullehrkräften in inklusiven Unterrichtssettings. Empirische Sonderpädagogik, 6 (1), 17-32.

Gebhardt, M., Schwab, S., Gmeiner, S., Ellmeier, B., Rossmann, P., \& Gasteiger Klicpera, B. (2013). Grazer Skala zur Lehrerkooperation im integrativen Unterricht (GSLK). Empirische Pädagogik, 27 (1), 5-21.

Gräsel, C., Fussangel, K., \& Pröbstel, C. (2006). Lehrkräfte zur Kooperation anregen - eine Aufgabe für Sisyphos? Zeitschrift für Pädagogik, 52 (2), 205-219.

Gurgur, H., \& Uzuner, Y. (2011). Examining the Implementation of Two Co-Teaching Models: Team Teaching and Station Teaching. International Journal of Inclusive Education, 15 (6), 589-610. https://doi.org/10.1080/13603110903265032.

Haeberlin, U., Jenny-Fuchs, E., \& Moser Opitz, E. (1992). Zusammenarbeit. Wie Lehrpersonen Kooperation zwischen Regel- und Sonderpädagogik in integrativen Kindergärten und Schulklassen erfahren. Bern: Haupt.

Heinrich, M., \& Lübeck, A. (2013). Hilflos häkelnde Helfer? Zur pädagogischen Rationalität von Integrationshelfer/inne/n im inklusiven Unterricht. Bildungsforschung, 10 (1), 91110.

Hellmich, F., Hoya, F., Görel, G., \& Schwab, S. (2017). Unter welchen Voraussetzungen kooperieren Grundschullehrkräfte im inklusiven Unterricht? Eine Studie zu den Bedingungen der Kooperationsbereitschaft von Grundschullehrerinnen und -lehrern im inklusiven Unterricht. Empirische Sonderpädagogik, 9 (1), 36-51.

Kreis, A., Wick, J., \& Kosorok Labhart, C. (2014). Wahrgenommene Zuständigkeiten von pädagogischem Personal in integrativen Schulen des Kantons Thurgau. Empirische Sonderpädagogik, 6 (4), 333-349.

Kreis, A., Wick, J., \& Kosorok Labhart, C. (2016). Aktivitätenrepertoires von Regellehrpersonen an inklusiven Schulen - eine Typologie. In V. Moser \& B. Lütje-Klose (Hrsg.), Schulische Inklusion (62. Beiheft der Zeitschrift für Pädagogik) (S. 140-159). Weinheim: Beltz Juventa.

Kullmann, H. (2010). Lehrerkooperation. Ausprägungen und Wirkungen am Beispiel des naturwissenschaftlichen Unterrichts an Gymnasien. Münster et al.: Waxmann.

Kunze, K. (2016). Multiprofessionelle Kooperation - Verzahnung oder Differenzierung? Einige Einwände gegen die Polarisierungstendenz einer Diskussion. In T.-S. Idel, F. Dietrich, K. Kunze, K. Rabenstein \& A. Schütz (Hrsg.), Professionsentwicklung und Schulstrukturreform. Zwischen Gymnasium und neuen Schulformen in der Sekundarstufe (S. 261-277). Bad Heilbrunn: Klinkhardt.

Laubenstein, D., Lindmeier, C., Guthöhrlein, K., \& Scheer, D. (2015). Auf dem Weg zur schulischen Inklusion. Empirische Befunde zum gemeinsamen Unterricht in rheinlandpfälzischen Schwerpunktschulen. Bad Heilbrunn: Klinkhardt.

Lütje-Klose, B., \& Urban, M. (2014). Professionelle Kooperation als wesentliche Bedingung inklusiver Schul- und Unterrichtsentwicklung. Teil 1: Grundlagen und Modelle inklusiver Kooperation. Vierteljahresschrift für Heilpädagogik und ihre Nachbargebiete, 83 (2), 112-123. https://doi.org/10.2378/vhn2014.art09d.

Marvin, C. (1990). Problems in School-Based Language Consultation and Collaboration Services: Defining the Terms and Improving the Process. In W. A. Secord (Hrsg.), Best Practices in School Speech-Language Pathology (S. 37-47). San Antonio, TX: The Psychological Corporation. 
Pool Maag, S., \& Moser Opitz, E. (2014). Inklusiver Unterricht - grundsätzliche Fragen und Ergebnisse einer explorativen Studie. Empirische Sonderpädagogik, 6 (2), 133-149.

Rolff, H.-G., \& Steinweg, A. (1980). Realität und Entwicklung von Lehrerkooperation. In H.-G. Rolff (Hrsg.), Soziologie der Schulreform. Theorien, Forschungsberichte, Praxisberatung (S. 113-129). Weinheim: Beltz.

Schwab, S. (2017). Interprofessionelle Lehrkraftkooperation im inklusiven Unterricht aus der Perspektive der Schülerinnen und Schüler. Unterrichtswissenschaft, 45 (4), 262 279.

Schwab, S., Gebhardt, M., \& Krammer, M. (2015). Umsetzung der schulischen Integration von SchülerInnen mit Behinderung(en) in Österreich. In D. Blömer, M. Lichtblau, A.-K. Jüttner, K. Koch, M. Krüger \& R. Werning (Hrsg.), Perspektiven auf inklusive Bildung. Gemeinsam anders lehren und lernen (S. 132-138). Wiesbaden: Springer VS. https://doi.org/10.1007/978-3-658-06955-1_19.

Scruggs, T. E., Mastropieri, M. M., \& McDuffie, K. A. (2007). Co-Teaching in Inclusive Classrooms. A Metasynthesis of Qualitative Research. Council for Exceptional Children, 73 (4), 392-416. https://doi.org/10.1177/001440290707300401.

Stefanidis, A., \& Strogilos, V. (2015). Union Gives Strength: Mainstream and Special Education Teachers' Responsibilities in Inclusive Co-Taught Classrooms. Educational Studies, 41 (4), 393-413. https://doi.org/10.1080/03055698.2015.1018872.

Steinert, B., Klieme, E., Maag Merki, K., Döbrich, P., Halbheer, U., \& Kunz, A. (2006). Lehrerkooperation in der Schule. Konzeption, Erfassung, Ergebnisse. Zeitschrift für Pädagogik, 52 (2), 185-204.

Stichweh, R. (1992). Professionalisierung, Ausdifferenzierung von Funktionssystemen, Inklusion. Betrachtungen aus systemtheoretischer Sicht. In B. Dewe, W. Ferchhoff \& F.-O. Radtke (Hrsg.), Erziehen als Profession. Zur Logik professionellen Handelns in pädagogischen Feldern (S. 36-48). Opladen: Leske + Budrich.

Vangrieken, K., Dochy, F., Raes, E., \& Kyndt, E. (2015). Teacher Collaboration: A Systematic Review. Educational Research Review, 15, 17-40. https://doi.org/10.1016/j.edurev. 2015.04.002.

Weiss, M. P., \& Lloyd, J. W. (2002). Congruence between Roles and Actions of Secondary Special Educators in Co-Taught and Special Education Settings. Journal of Special Education, 36 (2), 58-68. https://doi.org/10.1177/00224669020360020101.

Werning, R. (2014). Stichwort: Schulische Inklusion. Zeitschrift für Erziehungswissenschaft, 17, 601-623. https://doi.org/10.1007/s11618-014-0581-7.

Willmann, M. (2009). Lehrer-Kooperation. In G. Opp \& G. Theunissen (Hrsg.), Handbuch schulische Sonderpädagogik (S. 470-478). Bad Heilbrunn: Klinkhardt.

Tanja Lindacher, Dr., geb. 1969, wissenschaftliche Mitarbeiterin am Lehrstuhl für Bildungsinstitutionen und Schulentwicklung an der Erziehungswissenschaftlichen Fakultät der Universität Erfurt.

E-Mail: tanja.lindacher@uni-erfurt.de

Kathrin Dedering, Prof. Dr., geb. 1974, Inhaberin des Lehrstuhls für Bildungsinstitutionen und Schulentwicklung an der Erziehungswissenschaftlichen Fakultät der Universität Erfurt.

E-Mail: kathrin.dedering@uni-erfurt.de

Anschrift: Universität Erfurt, Erziehungswissenschaftliche Fakultät, Fachgebiet Schulpädagogik, Nordhäuser Str. 63, 99089 Erfurt 